\title{
Assessment of Functional Food Dioscorea Japonica Paste As A Thickened Liquid For Elderly With Dysphagia
}

\section{Yuka Konoike}

Okayama Prefectural University

Izumi Tsukayama

Okayama Prefectural University

Mei Oji

Okayama Prefectural University

Takayo Kawakami

Okayama Prefectural University

\section{Kayoko Ishii}

Fukuyama University

\section{Toshiko Suzuki-Yamamoto ( $\nabla$ toshiko@fhw.oka-pu.ac.jp )}

Okayama Prefectural University

\section{Research Article}

Keywords: Dysphagia diet, Dioscorea japonica paste, Thickened liquids, Viscosity, Food functionality

Posted Date: January 7th, 2022

DOI: https://doi.org/10.21203/rs.3.rs-1158149/v1

License: () (1) This work is licensed under a Creative Commons Attribution 4.0 International License. Read Full License 
1 Assessment of functional food Dioscorea japonica paste as a thickened liquid for

2 elderly with dysphagia

3

4 Yuka Konoike ${ }^{1,2}$, Izumi Tsukayama ${ }^{1}$, Mei Oji ${ }^{1,3}$, Takayo Kawakami ${ }^{1}$, Kayoko Ishii ${ }^{2}$,

5 and Toshiko Suzuki-Yamamoto ${ }^{1^{*}}$

6

$7 \quad{ }^{1}$ Department of Nutritional Science, Okayama Prefectural University, 111 Kuboki, Soja,

8 Okayama, 719-1197, Japan

$9 \quad{ }^{2}$ Department of Nutrition and Life Science, Fukuyama University, Sanzo, Gakuen-cho 1,

10 Fukuyama, Hiroshima, 729-0292, Japan

$11{ }^{3}$ The Faculty of Food Culture, Kurashiki Sakuyo University, 3515 Tamashimanagao,

12 Kurashiki, Okayama, 710-0292, Japan

14 *To whom correspondence should be addressed:

15 Toshiko Suzuki-Yamamoto

16 Department of Nutritional Science, Okayama Prefectural University, 111 Kuboki, Soja, 
17 Okayama, 719-1197, Japan

18 Tel: (81) 866-94-2156

19 Fax: (81) 866-94-2156

20 E-mail: toshiko@fhw.oka-pu.ac.jp 


\section{Abstract}

22 Background: In the elderly, reduced mastication and swallowing functions result in

23 malnutrition and deterioration in the quality of life. As individuals age in the society, the

24 novel concept of dysphagia diet is essential in order to prevent lifestyle and chronic

25 diseases and maintain nutrition intake. Recently, we reported that Dioscorea japonica, a

26 wild yam, has preventive effects on chronic inflammation via the inhibition of

27 proinflammatory lipid mediator synthesis. The paste of Dioscorea japonica showed

28 conformable physical properties as a thickened liquid for patients with dysphagia in

29 rheological analysis. In the present study, we focused on the unique physical properties

30 of Dioscorea japonica paste and evaluated its stability and usefulness as a thickened

31 liquid compared with commercially available thickened liquids.

32 Methods: The paste prepared using a uniformly freeze-dried Dioscorea japonica powder

33 could suitably modify the viscosity by altering the blending amount. Viscosities of the

34 Dioscorea japonica paste, xanthan gum, and commercially available thickened liquids

35 were measured using a cone and plate viscometer after 1 min by employing the following

36 setting: temperature of $20^{\circ} \mathrm{C}$ and shear rate of $50 \mathrm{~s}^{-1}$. The effect of changes in temperature 
37 and $\mathrm{pH}$, and addition of $\mathrm{NaCl}$ and $\alpha$-amylase, on viscosity was compared among the

38 thickened liquids.

39 Results: Compared with the other commercially available agents, the Dioscorea japonica

40 paste was stable in terms of viscosity on the addition of $\mathrm{NaCl}$, and no change was

41 observed on the addition of $\alpha$-amylase as similar as the others. Although the Dioscorea

42 japonica paste was relatively stable in terms of viscosity with change in $\mathrm{pH}$, it was slightly

43 unstable with change in temperature.

44 Conclusion: The findings of this study indicate that the Dioscorea japonica paste is

45 useful as a novel type of thickened liquid for patients with dysphagia.

46

47 Key words: Dysphagia diet, Dioscorea japonica paste, Thickened liquids, Viscosity,

$48 \quad$ Food functionality 


\section{Background}

50 With Japan on its way to becoming a super-aged society, patients with dysphagia are also

51 increasing [1-3]. Dysphagia will result in not only impaired quality of life and

52 malnutrition but also severe diseases such as aspiration pneumonia [4,5]. To maintain a

53 healthy body and activities of daily living in the elderly, receiving oral nutrition from

54 high quality food is essential. In patients with impaired swallowing function,

55 consumption of texture-modified food and thickened liquids is preferable [6]; therefore,

56 commercial thickening and gelling agents are commonly used. Today, xanthan gum-based

57 thickened liquids $[7,8]$ are mainly used to prepare foods that vary in texture and for

58 patients with dysphagia for ease of swallowing. Selecting an appropriate thickened liquid

59 to achieve desirable rheological properties based on the swallowing function of

60 individuals and avoid the risk of aspiration is crucial $[8,9]$. However, thickened liquids

61 with unsuitable viscosity are occasionally used in hospitals and welfare facilities. In Japan,

62 a standard framework for classifying dysphagia diets is in place, and the Japanese Society

63 of Dysphagia Rehabilitation developed the Japanese Dysphagia Diet 2013 (JDD2013)

64 [10]. JDD2013 classifies thickened liquids according to drinking properties, visual 
65 properties, and viscosity values as follows: Level 1, Mildly thick; Level 2, Moderately

66 thick; Level 3, Extremely thick. Watanabe et al. describes that JDD2013 defines these

67 thickened liquids as follows, mildly thick: flowing quickly with the tilting of a spoon,

68 leaving a thin trace of residue in the cup after being poured, and suitable for mild cases

69 of dysphagia; moderately thick: flowing slowly with the tilting of a spoon, leaving a film

70 of residue in the cup after being poured, and suitable for moderately severe cases of

71 dysphagia; extremely thick: maintaining most of its form with the tilting of a spoon, not

72 flowing quickly after being poured, and suitable for more severe cases of dysphagia

73 showing a risk of aspiration using moderately thick liquids [11].

74 The main causes of death in Japan are chronic diseases, such as malignant

75 neoplasms, heart diseases, and cerebrovascular diseases, which are associated with

76 chronic inflammation in many cases. Prevention of chronic inflammation is of utmost

77 importance to maintain a healthy and long-living society. We have been exploring food

78 functionality for the prevention of chronic inflammation by targeting the synthetic

79 pathway of proinflammatory lipid mediators, such as prostaglandin (PG) $\mathrm{E}_{2}$ and

80 leukotrienes, derived from $\omega-6$ arachidonic acid. In our previous study, we have 
81 demonstrated that Dioscorea japonica, a wild yam, suppresses the activity of

82 cyclooxygenase-2 (COX-2) and microsomal prostaglandin E (PGE) synthase-1, which

83 are key enzymes in the production of proinflammatory $\mathrm{PGE}_{2}$, and has anti-inflammation

84 and anti-carcinogenesis [12,13]. Recently, it is reported that diosgenin, a functional

85 ingredient of Dioscorea japonica, downregulates COX-2 expression selectively in

86 macrophages via glucocorticoid receptor and improves LPS-induced liver inflammation

87 [14].

88

Dioscorea japonica is a relative of the Dioscoreaceae family that is native to

89 Japan. The yam tubers of this species are usually considered edible and are beneficial for

90 nutritional fortification $[15,16]$. Moreover, they have been shown to have other beneficial

91 effects such as gastric mucosal protection and digestive enhancement. In some countries

including Japan, the wild yam has been used as folk medicine against asthma, rheumatoid

arthritis, bronchitis, and other diseases. Dioscoreaceae yam tubers have distinguishing

94 physical properties, and among them, Dioscorea japonica yams have the highest viscosity.

95 Previously, we elucidated about the rheological properties of Dioscorea japonica tuber

96 and its potential application in dysphagia diets [17]. The our previous study shows that 
97 Dioscorea japonica powder of $30 \%$ weight/volume (W/V) was suitable for all the items

98 of hardness, cohesiveness, and adhesiveness in the standard (level II for dysphagia diet)

99 of Texture Profile Analysis specified by the Ministry of Health, Labour and Welfare for

100 dysphagia diet [17]. In addition, the rheological analysis indicated that $20 \%-30 \%(\mathrm{~W} / \mathrm{V})$

101 of Dioscorea japonica powder showed a rheological profile similar to that of $0.2 \%$

102 xanthan gum as a true polymer solution, and [18] that it was more suitable as a thickened

103 liquid for dysphagia than grated raw Dioscorea japonica [18]. These results suggested

104 that Dioscorea japonica powder has potential application in dysphagia diets. Furthermore,

105 the physical property and swallowing compatibility of the paste of Dioscorea japonica

106 powder was similar to those of yogurt, which is often used to swallowing training for

107 patients with dysphagia, and the property of the paste was similar to that of xanthan gum

108 and commercially available thickened liquids in the study of texture analysis and

109 swallowing function evaluation [19]. These distinctive qualities of Dioscorea japonica in

110 terms of food functionality and physical properties could be beneficially applied in a

111 dysphagia diet of good quality. Moreover, whether its physical properties remain stable

112 during cooking and food processing will need to be investigated. In the present study, we 
113 compared the viscosity of Dioscorea japonica paste with that of xanthan gum and

114 commercially available thickened liquids under several conditions such as changes in

115 temperature and $\mathrm{pH}$, along with the addition of $\mathrm{NaCl}$ and $\alpha$-amylase.

\section{Methods}

\section{Sample preparation}

119 Dioscorea japonica powder was purchased from Naturalskyway Co. (Tokyo, Japan). It

120 was mixed with distilled water or $20 \mathrm{mM}$ sodium phosphate buffer $(\mathrm{pH} 7.0)$ at $20^{\circ} \mathrm{C} \pm 2^{\circ} \mathrm{C}$

121 using an electric mixer (bamixM250, ESGE Ltd, Mettlen, Switzerland) at room

122 temperature for $5 \mathrm{~min}$. The uniformly mixed Dioscorea japonica paste was kept as is for

$12320 \mathrm{~min}$ to remove any bubbles that were formed. Commercially available thickening

124 agent A (TROMELIN V) containing dextrin, thickening polysaccharide, and potassium

125 chloride was purchased from NUTRI Co., Ltd. (Yokkaichi, Japan); commercially

126 available thickening agent B (TSURURINKO Quickly) containing dextrin, xanthan gum,

127 calcium lactate, and trisodium citrate was purchased from CLINICO Co., Ltd. (Tokyo,

128 Japan); and xanthan gum was purchased from Neosoft XR, Taiyo Kagaku Co., Ltd. 
129 (Yokkaichi, Japan). They were added to distilled water or $20 \mathrm{mM}$ sodium phosphate

130 buffer $(\mathrm{pH} 7.0)$ at $20^{\circ} \mathrm{C} \pm 2^{\circ} \mathrm{C}$ and stirred manually with a spatula approximately 60

131 times/min for 1 min. Next, the thickened liquid $\mathrm{A}$ and $\mathrm{B}$ and liquid xanthan gum were

$132 \mathrm{kept}$ as is for 20 and $50 \mathrm{~min}$, respectively, at $20^{\circ} \mathrm{C} \pm 2^{\circ} \mathrm{C}$. Dioscorea japonica paste and the

133 thickened liquids were stirred manually 10 times before the viscosity analysis.

135 Viscosity analysis

136 Viscosity of the thickened liquids was analyzed according to the method established by

137 JDD2013 (the Japanese Society of Dysphagia Rehabilitation (JSDR) dysphagia diet 138 committee) [10], using a cone and plate viscometer (VISCOMETER TV-25; Toki Sangyo,

139 Co., Ltd., Tokyo, Japan) having a cone diameter and angle of $28 \mathrm{~mm}$ and $3^{\circ}$, respectively.

140 Viscosity of all thickened liquids was measured after 1 min under the following setting:

141 temperature of $20^{\circ} \mathrm{C}$ and shear rate of $50 \mathrm{~s}^{-1}$.

142

143 Line spread test

144 The line spread test (LST), another simple viscosity test established by JDD2013 [10], 
146 A hollow ring having an internal diameter of $30 \mathrm{~mm}$ and height of $28 \mathrm{~mm}$ was filled with

$14720 \mathrm{~mL}$ of the sample and was left to stand for $30 \mathrm{~s}$. The hollow ring was then lifted and

148 the sample was allowed to spread for $30 \mathrm{~s}$. The distance covered by the liquid along the

149 six axes were read and averaged [20]. This test was performed three times each, and each

150 value was averaged and evaluated.

152 Effect of temperature on the viscosity of the thickened liquids

153 The prepared samples were kept for 20 min at $10,20,40$, or $60^{\circ} \mathrm{C}$ before conducting the

154 viscosity analysis. All samples were adjusted to $150-300 \mathrm{mPa} \cdot \mathrm{s}$ as moderately thick

155 according to JDD2013 by the JSDR dysphagia diet committee [10].

156

157 Effect of pH on the viscosity of the thickened liquid

158 After preparation of the thickened liquids, $\mathrm{pH}$ of all samples was changed by addition of

159 the following buffers (final concentration of $20 \mathrm{mM}$ ): sodium acetate buffer $(\mathrm{pH} 4.0)$,

160 sodium phosphate buffer ( $\mathrm{pH} 7.0)$, and glycine buffer ( $\mathrm{pH} 9.0)$. 


\section{Assessment of $\alpha$-amylase resistance}

163 After preparation of the thickened liquids, they were incubated with $\alpha$-amylase $(1-1,000$

$164 \mu \mathrm{g} / \mathrm{mL}$, Wako Pure Chemical Industries, Ltd., Osaka, Japan) in $20 \mathrm{mM}$ sodium phosphate 165 buffer $(\mathrm{pH} 7.0)$ at $37^{\circ} \mathrm{C}$ for $10 \mathrm{~min}$. Potato starch liquid (5.3\%) was used for comparison.

166 Their viscosities were then measured at $20^{\circ} \mathrm{C}$ for $1 \mathrm{~min}$, as previously described.

\section{Statistics}

Data were statistically evaluated by ANOVA using Bonferroni test or Dunnett's test using a significance level of $p<0.01$.

172 Results

173 Correlation between concentration and viscosity of Dioscorea japonica paste and

\section{4 thickened liquids}

175 Several commercially available thickened liquids have individual physical properties,

176 with different viscosities and textures even at the same concentration [21,22]. The

177 correlation between concentration and viscosity of Dioscorea japonica paste, liquid 
178 xanthan gum, and commercially available thickened liquids A and B was confirmed (Fig.

179 1). Table 1 shows the comparative concentration of each thickener based on the three-

180 stage classification by established standard viscosity of JDD2013, "mildly thick,"

181 "moderately thick," and "extremely thick" [10]. Dioscorea japonica paste $(9.5 \%, 14 \%$,

182 and $17 \%)$, liquid xanthan gum $(0.5 \%, 1.4 \%$, and $2.0 \%)$, thickened liquid $\mathrm{A}(1.0 \%, 1.8 \%$,

183 and $2.5 \%)$, and thickened liquid B $(1.0 \%, 2.0 \%$, and 3.0\%) were prepared to the median

184 of each standard viscosity. Their viscosities and LST values were measured (Table 2) and

185 whether they matched with the criteria established by JDD2013 was evaluated. In the

186 subsequent experiments, the concentration adjusted to "moderately thick" was used.
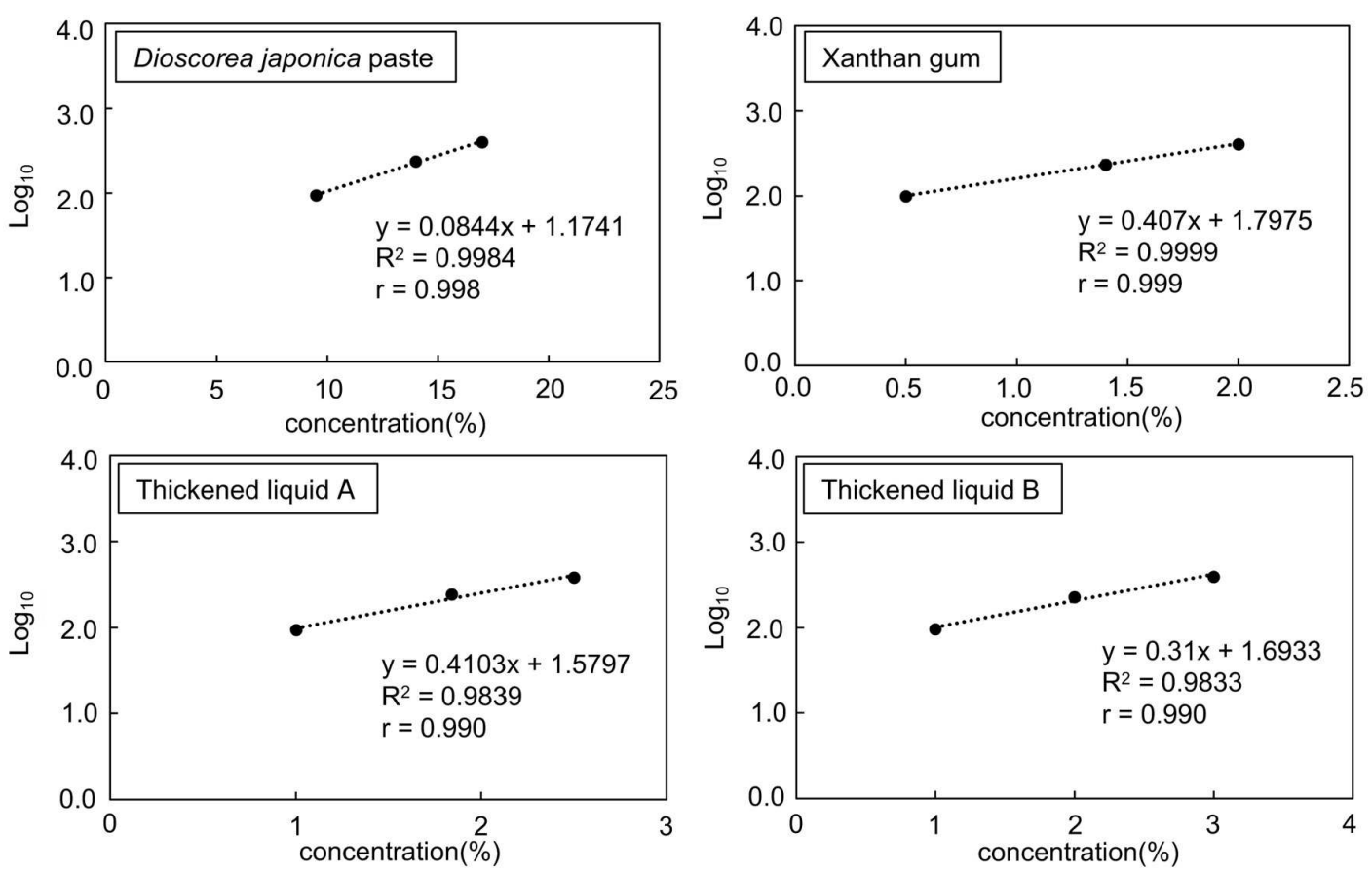

187

188 Fig. 1 Relationship between concentration and viscosity in each sample 
189 Dioscorea japonica paste was evaluated at concentrations of $9.5 \%, 14 \%$, and $17 \%$.

190 Xanthan gum was evaluated at concentrations of $0.5 \%, 1.4 \%$, and $2.0 \%$. Thickened liquid

191 A was evaluated at concentrations of $1.0 \%, 1.8 \%$, and $2.5 \%$. Thickened liquid B was

192 evaluated at concentrations of $1.0 \%, 2.0 \%$, and $3.0 \%$. The solutes were dissolved in

193 distilled water. The viscosity of all samples was assessed using a viscometer at $20^{\circ} \mathrm{C}$ after

194 five independent measurements. The viscosity is indicated as the values of common

195 logarithm.

196

197 Table 1 Sample concentration on each viscosity

\begin{tabular}{lcccc}
\hline Viscosity (mPa-s) & $\begin{array}{c}\text { Dioscorea } \\
\text { japonica paste } \\
(\%)\end{array}$ & $\begin{array}{c}\text { Xanthan gum } \\
(\%)\end{array}$ & $\begin{array}{c}\text { Thickened liquid A } \\
(\%)\end{array}$ & $\begin{array}{c}\text { Thickened liquid B } \\
(\%)\end{array}$ \\
\hline Mildly thick (50-150) & $6.22-11.87$ & $<0.93$ & $0.29-1.45$ & $0.02-1.56$ \\
Moderately thick (150-300) & $11.87-15.44$ & $0.93-1.67$ & $1.45-2.19$ & $1.56-2.53$ \\
Extremely thick (300-500) & $15.44-18.07$ & $1.67-2.21$ & $2.19-2.73$ & $2.53-3.24$ \\
\hline
\end{tabular}

198 According to Fig. 1, the concentration of each sample fitted to the standard viscosity was

199 determined. The solutes were dissolved in distilled water. The viscosity of all samples

200 was measured using a viscometer at $20^{\circ} \mathrm{C}$. Viscosity was categorized in three levels, as

201 established by the Japanese Dysphagia Diet 2013 (JDD2013) [10]. 
203 Table 2 The standard of viscosity and LST by Japanese Dysphagia Diet 2013 and 204 measured values

\begin{tabular}{lccc}
\hline & Mildly thick & Moderately thick & Extremely thick \\
\hline Standard viscosity $(\mathrm{mPa} \cdot \mathrm{s})[10]$ & $50-150$ & $150-300$ & $300-500$ \\
\hline Measured viscosity (mPa·s) & & & \\
\hline Dioscorea japonica paste & $92.96 \pm 0.10$ & $235.90 \pm 0.36$ & $401.84 \pm 0.93$ \\
Xanthan gum & $100.18 \pm 0.16$ & $236.12 \pm 0.64$ & $404.58 \pm 0.29$ \\
Thickened liquid A & $93.76 \pm 0.52$ & $242.28 \pm 0.35$ & $382.54 \pm 0.57$ \\
Thickened liquid B & $95.84 \pm 0.20$ & $229.92 \pm 0.22$ & $394.28 \pm 0.32$ \\
\hline Standard LST (mm) [10] & $36-43$ & $32-36$ & $30-32$ \\
\hline Measured LST (mm) & & & $31.39 \pm 0.06$ \\
\hline Dioscorea japonica paste & $39.11 \pm 0.93$ & $34.56 \pm 0.31$ & $31.56 \pm 0.56$ \\
xanthan gum & $39.39 \pm 0.24$ & $32.83 \pm 0.00$ & $31.39 \pm 0.24$ \\
thickened liquid A & $42.78 \pm 0.47$ & $34.33 \pm 0.48$ & $30.94 \pm 0.20$ \\
thickened liquid B & $42.06 \pm 0.29$ & $34.78 \pm 0.24$ & \\
\hline
\end{tabular}

205 Standard viscosity and LST categorized in three levels, as established by the Japanese

206 Dysphagia Diet 2013 (JDD2013) [10]. Dioscorea japonica paste was used at

207 concentrations of $9.5 \%, 14 \%$, and $17 \%$. Xanthan gum was used at concentrations of $0.5 \%$,

$2081.4 \%$, and 2.0\%. Thickened liquid A was used at concentrations of $1.0 \%, 1.8 \%$, and $2.5 \%$.

209 Thickened liquid B was used at concentrations of $1.0 \%, 2.0 \%$, and $3.0 \%$. The solutes 
210 were dissolved in distilled water. Viscosity of each sample was measured using a

211 viscometer at $20^{\circ} \mathrm{C}$ in five separate measurements. LST of each sample was measured

212 using a flat plate with concentric circles at $20^{\circ} \mathrm{C}$ after three independent measurements.

214 Effect of temperature change on the viscosity of thickened liquids

215 Changes in viscosity of the thickened liquids, Dioscorea japonica paste, liquid xanthan

216 gum, thickened liquid A, and thickened liquid B ("moderately thick," 229-242 mPa s)

217 were measured under various temperature conditions $\left(10,20,40\right.$, and $\left.60^{\circ} \mathrm{C}\right)$ (Fig. 2A).

218 With respect to temperature change, thickened liquid B was extremely stable without a

219 significant difference and liquid xanthan gum was relatively stable with increase less than

$22014 \%$ at each temperature. In contrast, the viscosity of Dioscorea japonica paste increased

221 by $16 \%$ at $10^{\circ} \mathrm{C}$ and decreased by approximately $20 \%$ at $40^{\circ} \mathrm{C}$ and $60^{\circ} \mathrm{C}$. Thickened liquid

222 A was more likely to be affected by change in temperature than Dioscorea japonica paste,

223 and the viscosity of thickened liquid $\mathrm{A}$ increased by $11 \%$ at $10^{\circ} \mathrm{C}$ and decreased by $22 \%$

224 and $54 \%$, respectively at $40^{\circ} \mathrm{C}$ and $60^{\circ} \mathrm{C}$. 
227 The effect of change in $\mathrm{pH}(\mathrm{pH} 4.0,7.0$, and 9.0) on the viscosity of the thickened liquids

228 was investigated (Fig. 2B). Thickened liquid A was stable under the testing pH conditions.

229 The viscosity of Dioscorea japonica paste marginally increased (within 7\%) at $\mathrm{pH} 4.0$

230 and 9.0 compared with $\mathrm{pH}$ 7.0. The viscosity of thickened liquid B showed a decrease of

$23116 \%$ at $\mathrm{pH} 9.0$. Among the viscosities of all thickened liquids, that of liquid xanthan gum

232 was the most unstable in terms of change in $\mathrm{pH}$ for $\mathrm{pH} 4.0$ and 9.0, showing significant

233 decreases of $30 \%$ at $\mathrm{pH} 4.0$ and $45 \%$ at $\mathrm{pH} 9.0$.

A.

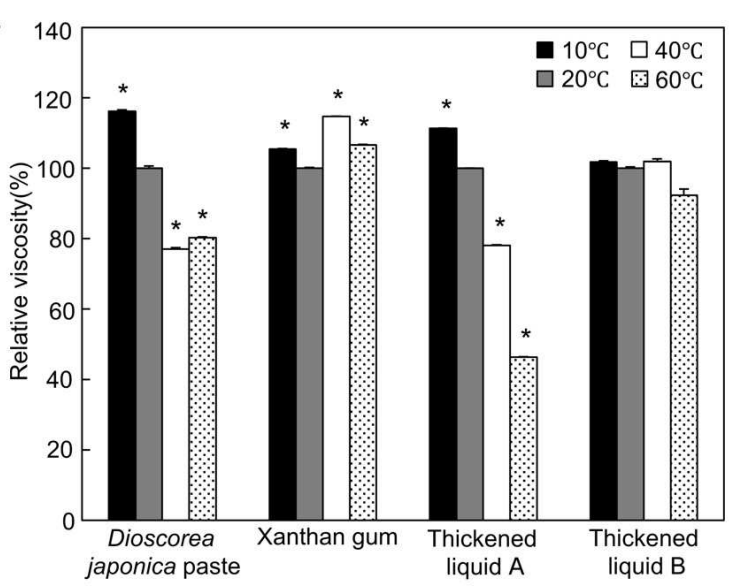

B.

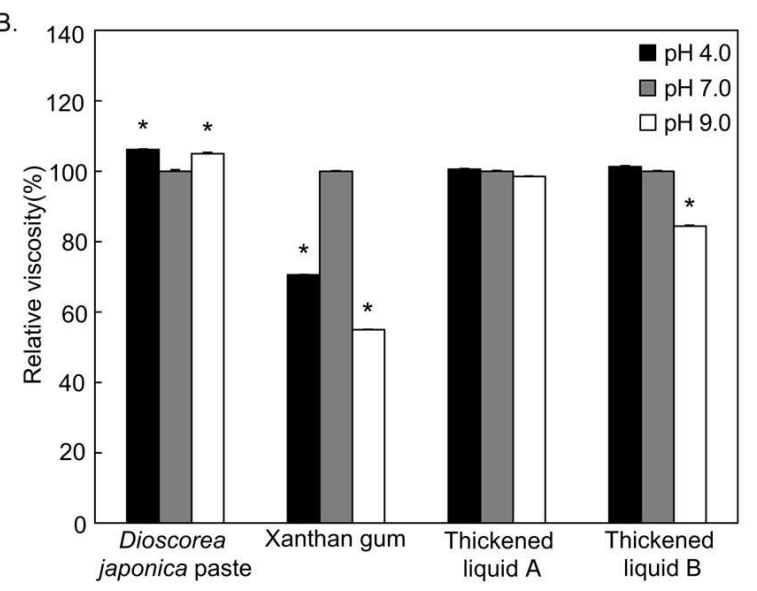

236 Fig. 2 Effects on stability after temperature and $\mathrm{pH}$ changes to viscosity for each sample

237 A: The viscosity of each sample was measured at $10,20,40$, and $60^{\circ} \mathrm{C}$ using a viscometer

238 after five independent measurements. The solutes were dissolved in distilled water. B: 
239 Each sample was prepared with sodium acetate buffer, sodium phosphate buffer, and

240 glycine buffer at $\mathrm{pH} 4.0,7.0$, and 9.0, respectively. Sample concentration: Dioscorea

241 japonica paste $12 \%$ (A) and $13.5 \%$ (B), xanthan gum $0.7 \%$, thickened liquid A $1.8 \%$,

242 thickened liquid B 2.1\%. The values of relative viscosities are means $\pm \mathrm{SE}$ represented as

243 relative values with respect to the amount at $20^{\circ} \mathrm{C}(\mathrm{A})$ or $\mathrm{pH}=7.0$ (B) as $100 \% .{ }^{*} p<0.01$

244 compared with $20^{\circ} \mathrm{C}(\mathrm{A})$ or $\mathrm{pH}=7.0(\mathrm{~B})$.

246 Effect of $\mathrm{NaCl}$ addition on the viscosity of thickened liquids

247 Because thickening agents are frequently added to foods that contain salt, the effect of

$248 \mathrm{NaCl}$ addition $(1 \%$ and $5 \%, \mathrm{~W} / \mathrm{V})$ on the viscosity of the thickened liquids was

249 investigated (Fig. 3A). A suitable $\mathrm{NaCl}$ concentration for soups or other relevant food

250 items is approximately $0.9 \%$, with $5 \% \mathrm{NaCl}$ usually considered as being outside the

251 normal accepted range. Among all the thickened liquids, the viscosity of Dioscorea

252 japonica paste was the most stable with $\mathrm{NaCl}$ addition, although its viscosity slightly

253 increased $(12 \%)$ with the addition of $5 \% \mathrm{NaCl}$. In contrast, the viscosities of liquid

254 xanthan gum and thickened liquids A and B significantly decreased approximately 50\% 
and $80 \%$ with the addition of $1 \%$ and $5 \% \mathrm{NaCl}$, respectively.

\section{Resistance of the viscosity of thickened liquids to $\alpha$-amylase addition}

258 Starch-based thickeners are also commonly used in the management of dysphagia;

259 however, the viscosity of such fluids can reduce on contact with saliva containing $\alpha-$

260 amylase. Therefore, at present, many commercially available thickening agents

261 demonstrate resistance to salivary digestion. To examine the direct effect of $\alpha$-amylase on

262 the viscosity of thickened liquids, the resistance of Dioscorea japonica paste to $\alpha$-amylase

263 was compared with the resistance of other thickened liquids to $\alpha$-amylase in the viscosity analysis (Fig. 3B). Addition of $\alpha$-amylase ( $1 \mu \mathrm{g} / \mathrm{mL}$ and $1 \mathrm{mg} / \mathrm{mL})$ significantly decreased the viscosity of potato starch liquid to $18 \%$ and $0.02 \%$, respectively, after $10 \mathrm{~min}$. In contrast, the viscosity of Dioscorea japonica paste was extremely resistant to $\alpha$-amylase,

267 which was similar to those observed for liquid xanthan gum and commercially available thickened liquids. 
A.

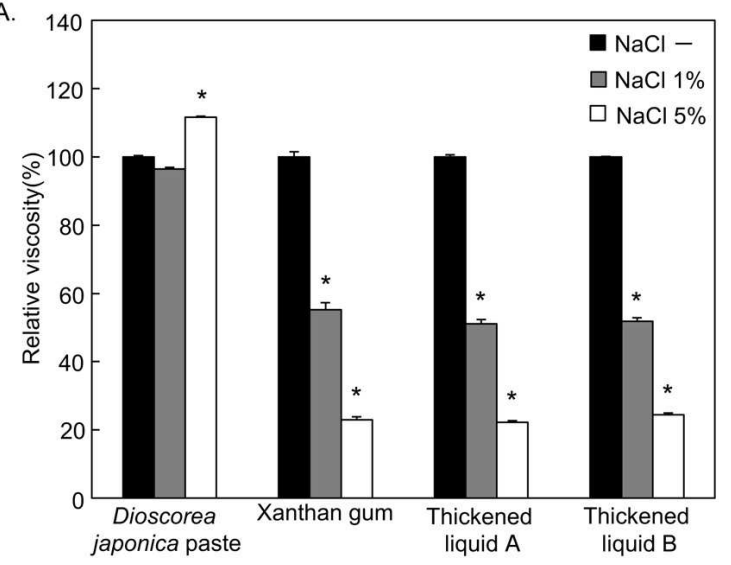

B.

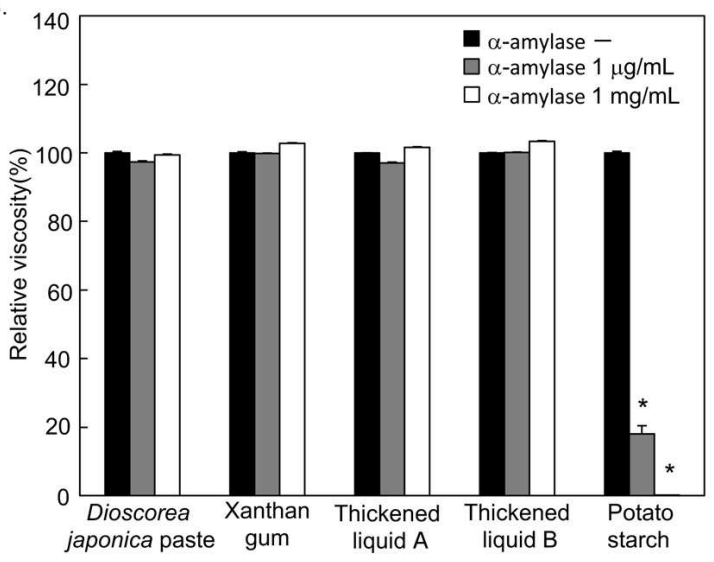

272 Fig. 3 Effects on stability after $\mathrm{NaCl}$ addition and resistance on $\alpha$-amylase activity to

273 viscosity of each sample

$274 \mathrm{~A}$ : In each sample $(\mathrm{pH}=7.0), 0 \%, 1 \%$, or $5 \% \mathrm{NaCl}$ in $20 \mathrm{mM}$ sodium phosphate buffer

was added. The viscosity of all samples was measured using a viscometer at $20^{\circ} \mathrm{C}$ after

five independent measurements. B: Potato starch was used at a concentration of $5.3 \%$. In

each sample or potato starch as a positive control, 0,1 , or $1,000 \mu \mathrm{g} / \mathrm{mL}$ of $\alpha$-amylase was

added. The solutes were dissolved in distilled water. The viscosity of all samples was

measured using a viscometer at $20^{\circ} \mathrm{C}$ after five independent measurements. Sample

concentration: Dioscorea japonica paste 15\% (A) and 12\% (B), xanthan gum $0.7 \%$,

281 thickened liquid A $1.8 \%$, thickened liquid B 2.1\%. The values of relative viscosity are 


\section{Discussion}

286 In the present study, the application of Dioscorea japonica paste as a thickened liquid was

287 investigated. Dioscorea japonica has multimodal functionality, which includes 288 prevention of chronic inflammation. In Japan, the most dominant standard for thickened

289 liquids is formulated by JDD2013 and categorized on the basis of viscosity. In this study,

290 we compared the usefulness of Dioscorea japonica paste as a thickened liquid with that

291 of commercially available thickening agents by viscosity analysis. The viscosity of

292 Dioscorea japonica paste was measured according to a measuring method suggested by

293 JDD2013; furthermore, variations in its viscosity were measured under various conditions.

294 The viscosity of Dioscorea japonica paste could be easily adjusted by altering its blending and temperature conditions, and its stability against changes in $\mathrm{pH}$ and $\alpha$-amylase activity

296 was similar to that of commercially available thickened liquids. In the presence of $\mathrm{NaCl}$,

297 the viscosity of commercial thickened liquids decreased significantly depending on the concentration, whereas that of Dioscorea japonica paste showed superior stability. Based on the above, the physical characteristics of Dioscorea japonica paste in the viscosity 
analysis were found to be exceptional, and Dioscorea japonica paste may be suitable as

301 a thickened liquid for patients with dysphagia. In recent years, xanthan gum-based thickened liquids, which are more stable than starch-based ones, have mainly been used [23]. The two types of thickened liquids used in this study (thickened liquid A and B) were also prepared using xanthan gum-based thickening agents. The two commercially available thickened liquids evaluated in this study mainly contain dextrin and a thickening polysaccharide, but their viscosity is believed to differ because the type and blending rate are different depending on the manufacturer [24,25]. Moreover, as with liquid xanthan gum and commercially available

309 thickened liquids, Dioscorea japonica paste displayed a positive correlation between the

310 added concentration and the common logarithmic value of viscosity, indicating that the

311 viscosity can be adjusted proportionally to the amount added. The thickened liquids were

312 then evaluated using the LST method and all the samples were matched with the criteria

313 of the LST value established by JDD2013 (Table 2). The LST method is a simple and

314 inexpensive viscosity measurement method for convenient instrument preparation that

315 has low variability within the experiments [20]. Although some aspects regarding the 
316 accuracy of the LST method should be taken into account [26], the LST values of the

317 Dioscorea japonica paste and commercially available thickened liquids investigated in

318 this study matched the JDD 2013 criteria as well as the viscosity assessment. Elderly people, particularly those who need meal assistance require more time to eat, and even if a thickening agent is added to warm foods to adjust the viscosity to an

321 appropriate level, the viscosity often changes before eating. Therefore, it is essential that

322 the viscosity of thickened liquids is stably maintained under conditions of change in

323 temperature. Deto et al. [24] reported that the viscosity of liquid xanthan gum does not

324 change significantly from $10^{\circ} \mathrm{C}$ to $90^{\circ} \mathrm{C}$ and therefore it is used as a raw material for

325 commercially available thickened liquids. Nevertheless, in the present study, the viscosity

326 showed the tendency to increase slightly at low or high temperatures (Fig. 2A). This is

327 thought to be due to a difference in determination method of physical property, i.e., our

328 viscosity analysis and their texture analysis. Hong et al. [7] reported that the viscosity of

329 xanthan gum-based thickened liquids is temperature-dependent. Indeed, in the present

330 study, the viscosity of the xanthan gum-based thickened liquid A showed a significant

331 decrease at high temperatures, whereas xanthan gum-based thickened liquid B was 
332 relatively stable in terms of viscosity in response to temperature changes. Although details

333 of the ingredients present in the commercially available thickening agents have not been

334 published, other ingredients have been added to some thickened liquids, such as thickened

335 liquid $\mathrm{B}$, to improve them so that their viscosity if not considerably affected change in

336 temperature. Compared with these thickened liquids, the Dioscorea japonica paste

337 showed a trend of having a slightly higher viscosity at low temperatures and a slightly

338 lower viscosity at high temperatures; however, this trend was not as significant as that

339 observed for commercially available thickened liquid A. The viscous component of yam,

340 including Dioscorea japonica, is derived from glycoproteins [27,28], and it has been

341 reported that proteins are denatured by heating and are insolubilized to decrease their

342 viscosity [29]. So the viscosity of Dioscorea japonica paste derived from the glycoprotein

343 moiety might be decreased at high temperatures in the present study.

344 Considering the stability of a thickened liquid, maintaining the viscosity of foods

345 and beverages even with changes in their $\mathrm{pH}$ is crucial. As shown in Fig. 2B, the viscosity

346 of Dioscorea japonica paste showed a slight increasing trend under acidic and alkaline

347 conditions (within $+7 \%$ ). In contrast, the viscosity of xanthan gum showed a considerable 
348 decrease of approximately $30 \%$ and $45 \%$ at $\mathrm{pH} 4.0$ and 9.0 , respectively, and that of

349 thickened liquid B showed decrease of approximately $15 \%$ at $\mathrm{pH} 9.0$. However, xanthan

350 gum-based thickened liquids have been reported to be tolerant to $\mathrm{pH}$ changes [30], and

351 so, we re-experimented with the same xanthan gum preparation method as that mentioned

352 in the reference [30]; however, the results did not show much difference with the present

353 study (data not shown). Furthermore, the effects of $\mathrm{pH}$ change varied for the two

354 commercially available xanthan gum-based agents. Yoon et al. [9] reported that $\mathrm{pH}$ has

355 different viscoelastic effects depending on the type of thickened liquids, and indeed, the

356 present study showed the differences among the xanthan gum and two commercially

357 available xanthan gum-based agents. By contrast, the viscosity of the Dioscorea japonica

358 paste was derived from glycoprotein and was shown to be relatively stable with change

359 in $\mathrm{pH}$.

361 soups, it is crucial that the viscosity is not considerably affected by the addition of salt.

362 In this study, changes in viscosity with the addition of $1 \%$ or $5 \% \mathrm{NaCl}$ was also evaluated 
commercially available thickened liquids decreased proportionally to the amount of $\mathrm{NaCl}$

365 added (Fig. 3A). The $\mathrm{NaCl}$ concentration of a typical soup is approximately $0.8 \%-0.9 \%$,

366 but even at $1 \% \mathrm{NaCl}$, which is close to the aforementioned value, a decrease in viscosity

367 of $40-\%-50 \%$ was observed in liquid xanthan gum and commercially available thickened

368 liquids. The viscosity of xanthan gum, which has a wide variety of structures with many

369 side chains surrounding the main chain [31], stabilizes after addition of salt at low

370 concentrations ( $1 \%$ or less) [32,33]. However, the present study showed that addition of

$3711 \%$ or more $\mathrm{NaCl}$ caused a decrease in the viscosity of liquid xanthan gum, indicating

372 that the thickening effect of xanthan gum was affected by $\mathrm{NaCl}$ concentration of the food.

373 In addition, it is known that the addition of $\mathrm{NaCl}$ has a notable effect on the rheological

properties of xanthan gum-based thickened liquids [34]. Analysis of the viscosity $2 \mathrm{~h}$ and

$3754 \mathrm{~h}$ after the addition of $\mathrm{NaCl}$ showed little difference from the present data of Fig.3A

376 (data not shown). Although the viscosity of the Dioscorea japonica paste increased by

377 approximately $10 \%$ with the addition of $5 \% \mathrm{NaCl}$, it showed exceptional stability

378 compared with the commercially available thickened liquids; moreover, the Dioscorea 
concentration of a typical soup). In previous study of tsukuneimo, ichoimo, and nagaimo,

381 which are types of wild yams, addition of more than $0.1 \mathrm{M}(\fallingdotseq 0.58 \%, \mathrm{~W} / \mathrm{V}) \mathrm{NaCl}$

382 decreased their viscosity by more than $20 \%$ [29]. These results were different in the case

383 of Dioscorea japonica paste, but the content of the viscous component may individually

384 vary among the yam family [28]. In particular, a stability of the viscosity of Dioscorea

385 japonica paste against addition of $\mathrm{NaCl}$ is superior physical properties, and it may be

386 useful in cooking and food processing of swallowing-adjusted foods.

Salivary $\alpha$-amylase hydrolyzes $\alpha$-1,4-glycosidic bonds of polysaccharides, such

as starch, and immediately decreases the viscosity of food and liquid thickened by starch.

389 However xanthan gum, whose main chain is composed of $\beta-1,4$-glycosidic bonds, and

xanthan gum-based commercial thickened liquids are resistant to $\alpha$-amylase, and their

viscosity does not change with the addition of $\alpha$-amylase. Although the viscous 
397 liquids, each liquid was found to have unique characteristics. However, even after

398 summarizing these characteristics, the Dioscorea japonica paste showed usefulness that

399 was comparable to that of commercially available thickened liquids.

\section{Conclusion}

402 Our previous study demonstrated that Dioscorea japonica suppressed $\mathrm{PGE}_{2}$ synthetic

403 pathway, which is associated with acute/chronic inflammation [12-14], and additionally

404 had potential applicability in the development of dysphagia diets [17,19]. In the present study, we demonstrated the usefulness of Dioscorea japonica paste as a thickened liquid, which has excellent suitability compared with commercial thickened liquids. In addition,

407 Dioscorea japonica showed high versatility because the viscosity of Dioscorea japonica

408 paste could be easily adjusted by modifying its blending amount and temperature.

409 Therefore, we believe that Dioscorea japonica paste would be a novel thickened liquid

410 with adequate food functionality for the prevention of proinflammatory lipid mediators-

411 related diseases. 
413 Abbreviations

414 JDD2013: Japanese Dysphagia Diet 2013; PGE 2 : prostaglandin E2; W/V: weight/volume;

415 JSDR: Japanese Society of Dysphagia Rehabilitation; LST: line spread test

416

417 Acknowledgments

418 This work was supported by JSPS KAKENHI (grant number 18K11134), Suzuken

419 Memorial Foundation, and Mitsui Sumitomo Insurance Welfare Foundation to T.S.-Y.

420 We thank Enago (www.enago.jp) for English language review.

421

422 Authors' contributions

423 Y.K. performed the experiments, analyzed data, and drafted the manuscript. I.T. and M.O.

424 prepared samples and conducted viscosity measurement. T.K. and K.I. conceptualized the

425 study and interpreted data. T.S-Y. supervised the work, conceived and designed the

426 studies, interpreted the data, and drafted the manuscript. All authors read and approved

427 the final manuscript. 
$429 \quad$ Funding

430 JSPS KAKENHI (grant number 18K11134), Suzuken Memorial Foundation (Nagoya,

431 Japan), Mitsui Sumitomo Insurance Welfare Foundation (Tokyo, Japan), and Wesco

432 Scientific Promotion Foundation (Okayama, Japan) to Toshiko Suzuki-Yamamoto.

433

434 Availability of data and materials

435 The datasets used in the current study are available from the corresponding author upon

436 reasonable request.

437

438 Ethics approval and consent to participate

439 All experiments complied with current laws of Japan.

440

441 Consent for publication

442 Not applicable.

443

444 Competing interests 
445 The authors have no competing interests to declare.

446

447 Author details

$448{ }^{1}$ Department of Nutritional Science, Okayama Prefectural University, 111 Kuboki, Soja,

449 Okayama, 719-1197, Japan

$450 \quad{ }^{2}$ Department of Nutrition and Life Science, Fukuyama University, Sanzo, Gakuen-cho 1, 451 Fukuyama, Hiroshima, 729-0292, Japan

$452{ }^{3}$ The Faculty of Food Culture, Kurashiki Sakuyo University, 3515 Tamashimanagao,

453 Kurashiki, Okayama, 710-0292, Japan

454

455 References

456 1. Sura L, Madhavan A, Carnaby G, Crary MA. Dysphagia in the elderly: management

457 and nutritional considerations. Clin Interv Aging. 2012;7:287-98.

$458 \quad$ http://dx.doi.org/10.2147/CIA.S23404.

459 2. Azzolino D, Damanti S, Bertagnoli L, Lucchi T, Cesari M. Sarcopenia and

$460 \quad$ swallowing disorders in older people. Aging Clin Exp Res. 2019;31:799-805. 
3. Khan A, Carmona R, Traube M. Dysphagia in the elderly. Clin Geriatr Med. https://doi.org/10.2340/16501977-0415.

5. Baine WB, Yu W, Summe JP. Epidemiologic trends in the hospitalization of elderly Medicare patients for pneumonia, 1991-1998. Am J Public Health. 2001;91:1121-3. https://doi.org/10.2105/ajph.91.7.1121.

6. Penman JP, Thomson M. A review of the textured diets developed for the management of dysphagia. J Hum Nutr Diet. 1998;11:51-60. https://doi.org/10.1046/j.1365-277X.1998.00079.x. 
477 food thickeners used for diet modification of patients with dysphagia: effect of

478 concentration. Dysphagia. 2013;28:205-11. https://doi.org/10.1007/s00455-012-

479 9433-x.

480

9. Yoon S-N, Yoo B. Effect of pH on Rheological Properties of Dysphagia-Oriented

Thickened Water. Prev Nutr food Sci. 2016;21:73-7.

482 http://dx.doi.org/10.3746/pnf.2016.21.1.73.

10. The dysphagia diet committee of the Japanese Society of Dysphagia Rehabilitation. The Japanese Dysphagia diet 2013. Jpn J Dysphagia Rehabil. 2013;17:255-67.

11. Watanabe E, Yamagata Y, Fujitani J, Fujishima I, Takahashi K, Uyama R, et al. The Criteria of Thickened Liquid for Dysphagia Management in Japan. Dysphagia. 2017;33:26-32. https://doi.org/10.1007/s00455-017-9827-x.

12. Suzuki-Yamamoto T, Tanaka S, Tsukayama I, Takafuji M, Hanada T, Arakawa T, 
494 involving down-regulation of prostaglandin $\mathrm{E}_{2}$ synthetic pathway. J Clin Biochem

495 Nutr. 2018;62:139-47. https://doi.org/10.3164/jcbn.17-54.

496 14. Tsukayama I, Mega T, Hojo N, Toda K, Kawakami Y, Takahashi Y, et al.

497 Diosgenin suppresses COX-2 and mPGES-1 via GR and improves LPS-induced liver

498 injury in mouse. Prostaglandins Other Lipid Mediat. 2021;156:106580.

499 https://doi.org/10.1016/j.prostaglandins.2021.106580.

500 15. Wanasundera JPD, Ravindran G. Nutritional assessment of yam (Dioscorea alata)

501 tubers. Plant Foods Hum Nutr. 1994;46:33-9. https://doi.org/10.1007/BF01088459.

502 16. Wanasundera JPD, Ravindran G. Effects of cooking on the nutrient and antinutrient

503 contents of yam tubers (Dioscorea alata and Dioscorea esculenta). Food Chem.

504 1992;45:247-50. https://doi.org/10.1016/0308-8146(92)90155-U.

505 17. Suzuki-Yamamoto T, Nomura N, Yamamoto S, Tanaka M, Mega T, Tsukayama I,

506 et al. Rheological analysis of Dioscorea japonica to dysphagia diet development for

507 the elderly. Bull factulty Heal Welf Sci Okayama Prefect Univ. 2015;22:47-56.

$508 \quad$ http://doi.org/10.15009/00001297. 
510 between the rheological properties of thickener solutions and their velocity through

511 the pharynx as measured by the ultrasonic pulse Doppler method. Biosci Biotechnol

512 Biochem. 2010;74:1598-605. https://doi.org/10.1271/bbb.100192.

513 19. Tanaka M, Tsukayama I, Yamamoto T, Nakamura T. Applicability of Swallowing

514 Sounds and Electromyography for Assessing the Ease of Swallowing of Foods.

515 Japan Soc Nutr food Sci. 2020;93-101. https://doi.org/10.4327/jsnfs.73.93.

516 20. Mann LL, Wong K. Development of an objective method for assessing viscosity of

517 formulated foods and beverages for the dysphagic diet. J Am Diet Assoc.

518 1996;96:585-8. https://doi.org/10.1016/S0002-8223(96)00160-5.

519 21. Garcia JM, Chambers EI, Matta Z, Clark M. Viscosity measurements of nectar- and

520 honey-thick liquids: product, liquid, and time comparisons. Dysphagia.

$521 \quad 2005 ; 20: 325-35$. https://doi.org/10.1007/s00455-005-0034-9. 
23. Deto A, Egashira F, Kayashita J. Actual situation of commercial thickening agents for dysphagia in hospital and senior citizen institution. Bull factulty Heal Welf Sci Prefect Univ Hiroshima. 2008;3:33-42.

24. Deto A, Yamagata Y, Kayashita J. Effect of temperature on the physical properties Prefect Univ Hiroshima. 2007;2:39-47. commercial thickeners on the basis of their effects on the physical properties of https://doi.org/10.5264/eiyogakuzashi.70.59. study. Ann Rehabil Med. 2014;38:94-100. https://doi.org/10.5535/arm.2014.38.1.94. 

(Dioscorea opposita THUNB.). Japan Assoc Food Preserv Sci. 2003;29:229-36.

$544 \quad$ https://doi.org/10.5891/jafps.29.229. https://doi.org/10.5891/jafps.26.3.

30. Hadde EK, Nicholson TM, Cichero JAY. Rheological characterisation of thickened Mucilage, and effects of treatments on viscosity from Yam (Dioscorea oppossita Thunb.). Japan Assoc Food Preserv Sci. 2000;26:3-9. 85. http://dx.doi.org/10.1108/NFS-06-2014-0053. https://doi.org/10.11541/jag1994.43.385. 
33. Sato T, Takashi N, Fujita H. Double-stranded Helix of Xanthan: Dimensional and

559 Hydrodynamic Properties in 0.1 M Aqueous Sodium Chloride. Macromolecules.

560 1984;17:2696-700. https://doi.org/10.1021/ma00142a043.

561 34. Cho H-M, Yoo W, Yoo B. Effect of NaCl Addition on Rheological Behaviors of

562 Commercial Gum-Based Food Thickener Used for Dysphagia Diets. Prev Nutr food

563 Sci. 2015;20:137-42. http://dx.doi.org/10.3746/pnf.2015.20.2.137.

564

565 


\section{Figure legends}

567 Fig. 1 Relationship between concentration and viscosity in each sample

568 Dioscorea japonica paste was evaluated at concentrations of $9.5 \%, 14 \%$, and $17 \%$.

569 Xanthan gum was evaluated at concentrations of $0.5 \%, 1.4 \%$, and $2.0 \%$. Thickened liquid

570 A was evaluated at concentrations of $1.0 \%, 1.8 \%$, and $2.5 \%$. Thickened liquid B was

571 evaluated at concentrations of $1.0 \%, 2.0 \%$, and $3.0 \%$. The solutes were dissolved in

572 distilled water. The viscosity of all samples was assessed using a viscometer at $20^{\circ} \mathrm{C}$ after

573 five independent measurements. The viscosity is indicated as the values of common

574 logarithm.

576 Fig. 2 Effects on stability after temperature and $\mathrm{pH}$ changes to viscosity for each sample

577 A: The viscosity of each sample was measured at $10,20,40$, and $60^{\circ} \mathrm{C}$ using a viscometer

578 after five independent measurements. The solutes were dissolved in distilled water. B:

579 Each sample was prepared with sodium acetate buffer, sodium phosphate buffer, and

580 glycine buffer at $\mathrm{pH} 4.0,7.0$, and 9.0, respectively. Sample concentration: Dioscorea

581 japonica paste $12 \%(\mathrm{~A})$ and $13.5 \%(\mathrm{~B})$, xanthan gum $0.7 \%$, thickened liquid A $1.8 \%$, 
582 thickened liquid B 2.1\%. The values of relative viscosities are means \pm SE represented as

583 relative values with respect to the amount at $20^{\circ} \mathrm{C}(\mathrm{A})$ or $\mathrm{pH}=7.0(\mathrm{~B})$ as $100 \% .{ }^{*} p<0.01$

584 compared with $20^{\circ} \mathrm{C}(\mathrm{A})$ or $\mathrm{pH}=7.0(\mathrm{~B})$.

586 Fig. 3 Effects on stability after $\mathrm{NaCl}$ addition and resistance on $\alpha$-amylase activity to

587 viscosity of each sample

588 A: In each sample $(\mathrm{pH}=7.0), 0 \%, 1 \%$, or $5 \% \mathrm{NaCl}$ in $20 \mathrm{mM}$ sodium phosphate buffer

589 was added. The viscosity of all samples was measured using a viscometer at $20^{\circ} \mathrm{C}$ after

590 five independent measurements. B: Potato starch was used at a concentration of $5.3 \%$. In

591 each sample or potato starch as a positive control, 0,1 , or $1,000 \mu \mathrm{g} / \mathrm{mL}$ of $\alpha$-amylase was

592 added. The solutes were dissolved in distilled water. The viscosity of all samples was

measured using a viscometer at $20^{\circ} \mathrm{C}$ after five independent measurements. Sample

concentration: Dioscorea japonica paste 15\% (A) and 12\% (B), xanthan gum $0.7 \%$,

595 thickened liquid A $1.8 \%$, thickened liquid B 2.1\%. The values of relative viscosity are

596 means $\pm \mathrm{SE}$ represented as relative values with respect to the amount without $\mathrm{NaCl}(\mathrm{A})$

597 or $\alpha$-amylase (B). ${ }^{*} p<0.01$ compared with $0 \% \mathrm{NaCl}(\mathrm{A})$ or $0 \mu \mathrm{g} / \mathrm{ml} \alpha$-amylase (B). 
599 Table 1 Sample concentration on each viscosity

\begin{tabular}{lcccc}
\hline Viscosity (mPa·s) & $\begin{array}{c}\text { Dioscorea } \\
\text { japonica paste } \\
(\%)\end{array}$ & $\begin{array}{c}\text { Xanthan gum } \\
(\%)\end{array}$ & $\begin{array}{c}\text { Thickened liquid A } \\
(\%)\end{array}$ & $\begin{array}{c}\text { Thickened liquid B } \\
(\%)\end{array}$ \\
\hline Mildly thick (50-150) & $6.22-11.87$ & $<0.93$ & $0.29-1.45$ & $0.02-1.56$ \\
Moderately thick (150-300) & $11.87-15.44$ & $0.93-1.67$ & $1.45-2.19$ & $1.56-2.53$ \\
Extremely thick (300-500) & $15.44-18.07$ & $1.67-2.21$ & $2.19-2.73$ & $2.53-3.24$ \\
\hline
\end{tabular}

600 According to Fig. 1, the concentration of each sample fitted to the standard viscosity was

601 determined. The solutes were dissolved in distilled water. The viscosity of all samples

602 was measured using a viscometer at $20^{\circ} \mathrm{C}$. Viscosity was categorized in three levels, as

603 established by the Japanese Dysphagia Diet 2013 (JDD2013) [10].

604

605

606

607

608

609 
610 Table 2 The standard of viscosity and LST by Japanese Dysphagia Diet 2013 and

611 measured values

\begin{tabular}{lccc}
\hline & Mildly thick & Moderately thick & Extremely thick \\
\hline Standard viscosity $(\mathrm{mPa} \cdot \mathrm{s})[10]$ & $50-150$ & $150-300$ & $300-500$ \\
\hline Measured viscosity $(\mathrm{mPa} \cdot \mathrm{s})$ & & & \\
\hline Dioscorea japonica paste & $92.96 \pm 0.10$ & $235.90 \pm 0.36$ & $401.84 \pm 0.93$ \\
Xanthan gum & $100.18 \pm 0.16$ & $236.12 \pm 0.64$ & $404.58 \pm 0.29$ \\
Thickened liquid A & $93.76 \pm 0.52$ & $242.28 \pm 0.35$ & $382.54 \pm 0.57$ \\
Thickened liquid B & $95.84 \pm 0.20$ & $229.92 \pm 0.22$ & $394.28 \pm 0.32$ \\
\hline Standard LST (mm) [10] & $36-43$ & $32-36$ & $30-32$ \\
\hline Measured LST (mm) & & & $31.39 \pm 0.06$ \\
\hline Dioscorea japonica paste & $39.11 \pm 0.93$ & $34.56 \pm 0.31$ & $31.56 \pm 0.56$ \\
xanthan gum & $39.39 \pm 0.24$ & $32.83 \pm 0.00$ & $31.39 \pm 0.24$ \\
thickened liquid A & $42.78 \pm 0.47$ & $34.33 \pm 0.48$ & $30.94 \pm 0.20$ \\
thickened liquid B & $42.06 \pm 0.29$ & $34.78 \pm 0.24$ & \\
\hline
\end{tabular}

612 Standard viscosity and LST categorized in three levels, as established by the Japanese

613 Dysphagia Diet 2013 (JDD2013) [10]. Dioscorea japonica paste was used at

614 concentrations of $9.5 \%, 14 \%$, and $17 \%$. Xanthan gum was used at concentrations of $0.5 \%$,

$6151.4 \%$, and 2.0\%. Thickened liquid A was used at concentrations of $1.0 \%, 1.8 \%$, and $2.5 \%$.

616 Thickened liquid B was used at concentrations of $1.0 \%, 2.0 \%$, and $3.0 \%$. The solutes

617 were dissolved in distilled water. Viscosity of each sample was measured using a 
618 viscometer at $20^{\circ} \mathrm{C}$ in five separate measurements. LST of each sample was measured

619 using a flat plate with concentric circles at $20^{\circ} \mathrm{C}$ after three independent measurements. 